\title{
Ureteral reconstruction using a tapered non-vascularized bladder graft: an experimental study in a canine animal model
}

\author{
Lujia Zou', Shanhua Mao ${ }^{\dagger}$, Shenghua Liu, Limin Zhang, Tian Yang, Yun Hu, Qiang Ding and Haowen Jiang ${ }^{*}$
}

\begin{abstract}
Background: Reconstruction of ureteral defects and strictures remains problematic for urologists. We aimed to investigate the possibility of a tapered non-vascularized bladder graft as a novel substitute for ureteral reconstruction.

Methods: This experimental study was conducted on nine beagles. Under general anesthesia, a full-thickness graft with 5-6 cm in length was disassociated from the anterior upper wall of the bladder, and tapered into $1 / 3$ to $1 / 2$ thickness, remaining the urothelial surface. After removal of $5 \mathrm{~cm}$ of right-sided mid-ureter, the tapered bladder graft was tubularized along the long axis and then respectively anastomosed to the upper and lower stumps of the ureter. A retrograde urography through a cystostomy was performed 8 weeks after the ureteral reconstruction. The animals were euthanized, and histopathologic examinations of the neoureters were performed.

Results: There were no severe complications during postoperative follow-up. The urography indicated patent urine excretion and no fistula or stenosis. Histopathologic examinations of the neoureters showed open lumen with urothelial lining. Nutrient vessels were observed in healthy submucosa, lamina muscularis and peripheral connective tissue.

Conclusions: Our study implied that ureteral reconstruction by a tapered non-vascularized bladder graft was anatomically possible in our animal model. Further studies are expected to confirm long-term and functional outcomes.
\end{abstract}

Keywords: Animal model, Bladder graft, Non-vascularized, Reconstruction, Ureter

\section{Background}

Ureteral injuries usually arise from traumatic or iatrogenic causes [1]. In most cases, an end-to-end anastomosis without additional material can be applied for management of a short-segment ureteral defect or stricture. Furthermore, several classical surgical reconstructive methods have been well recognized, including ureteroplasty using a bladder muscle flap (Boari flap), ileal ureteral substitution, and renal autotransplantation [2].

\footnotetext{
* Correspondence: huashanurologyhwj@163.com

${ }^{\dagger}$ Equal contributors

Department of Urology, Huashan Hospital, Fudan University, No.12 Wulumuqi Middle Road, Shanghai 200040, People's Republic of China
}

However, these traditional methods have limitations in practical application. The Boari flap can reconstruct a limited length of distal or mid-ureter [3]. The ileal substitution has a variety of postoperative complications due to the characteristics of intestinal mucosa, including reabsorption of ammonium [4-6] and mucus secretion that may subsequently induce urinary tract infection and lithogenesis [5, 6]. Renal autotransplantation calls for a complicated procedure and is associated with possible infection and loss of renal function [1]. The Monti and Mitrofanoff techniques have been successfully developed to decrease postoperative intestinal complications, while intestinal involvement may still be related to leakage and ileus [7-9]. Concerning reconstructive materials, a 
bladder flap (or graft) with urothelial lining, which is similar to the ureter, seems ideal for ureteral replacement. We hypothesized that it may be possible to use a non-vascularized bladder graft for reconstruction of upper or mid-ureter. The primary concern was whether the bladder graft was able to survive without a vessel pedicle.

The main objective of our study was to investigate the possibility of a novel method using a tapered nonvascularized bladder graft to reconstruct the ureter in a canine animal model.

\section{Methods}

This experimental study was conducted on 9 healthy male beagles (approximately 1-year-old), which were obtained from School of Agriculture, Shanghai Jiao Tong University. The mean weight of the beagles at operation time was $10.6 \mathrm{~kg}$ (range $8.4-12.2 \mathrm{~kg}$ ). The dogs were housed individually in the stainless-steel cages in a controlled environment. Filtered water and a standard animal diet were available ad libitum. The study was carried out at the laboratory animal unit in School of Pharmacy, Fudan University during March to August, 2015.

The right ureter was chosen for the experimental procedures, while the left side was reserved as control. The surgical field was prepared and sterilized with povidoneiodine. Then a prophylactic dose of cefazolin was applied intramuscularly. The procedure was performed under general endotracheal anesthesia. The anesthesia was induced through intravenous administration of propofol $(4 \mathrm{mg} / \mathrm{kg})$, lidocaine $(1 \mathrm{mg} / \mathrm{kg})$, and diazepam $(0.3 \mathrm{mg} /$ $\mathrm{kg}$ ) and maintained with isoflurane in $100 \%$ oxygen. The laparotomy was conducted through a full-length midline abdominal incision. Abdominal viscera were inspected to exclude any possible abnormalities, particularly of the urinary system.

A rectangular region was marked by four loose ligations at each vertex on the anterior upper wall of the filling bladder, the volume of which was approximately 30 $40 \mathrm{~mL}$. The region was designed with $5-6 \mathrm{~cm}$ in length and $1 \mathrm{~cm}$ in width. After full thickness resection, the bladder graft was temporarily preserved in normal saline and gently squeezed until it turned pale.

Through the cystostomy, a 4.7-Fr ureteral stent was retrogradely inserted into the right ureter from the ureteral orifice. The right ureter was mobilized from the retroperitoneal tissue, and then approximately $5 \mathrm{~cm}$ was removed from the middle part. The non-vascularized bladder graft was tapered carefully by an ophthalmic scissor into $1 / 3$ to $1 / 2$ thickness $(2-3 \mathrm{~mm}$ ), keeping the urothelial surface intact. Thereafter, the tapered bladder graft was tubularized along the long axis, with the stent inside. The two ends of the graft tube were respectively anastomosed to the upper and lower stumps of the ureter by four sutures. (Figs. 1 and 2) After the anastomoses were checked in case of hemorrhage and urine leakage, the abdominal fascia was closed by 3-4 sutures. The surgical wound was then closed layer by layer.

All animals received cefazolin and flurbiprofen for the next 3 days and cefazolin for an additional 4 days. Specific signs were monitored twice a day, including temperature, respiratory rate, pulse, appetite, activity, defecation and urination. The surgical wound was observed and sterilized at the time of monitoring. Liquid diet was started a few hours after the procedure, and it was advanced to regular diet the next day. The ureteral stent was maintained for the postoperative 6 weeks and removed through a brief cystostomy. 8 weeks after the reconstructive procedure, a retrograde urography by a 5-Fr tube was performed through another cystostomy to assess the possibility of leakage or stenosis.

The animals were then euthanized by intravenous injection of potassium chloride under general anesthesia. After thorough exploration of the abdominal cavity and surgical field, the right-sided kidney, ureter (together with peripheral connective tissue) and part of the bladder were resected and fixed with formalin immediately. The specimens were embedded with paraffin and stained with hematoxylin \& eosin. Two experienced genitourinary pathologists separately finished the microscopic examination.

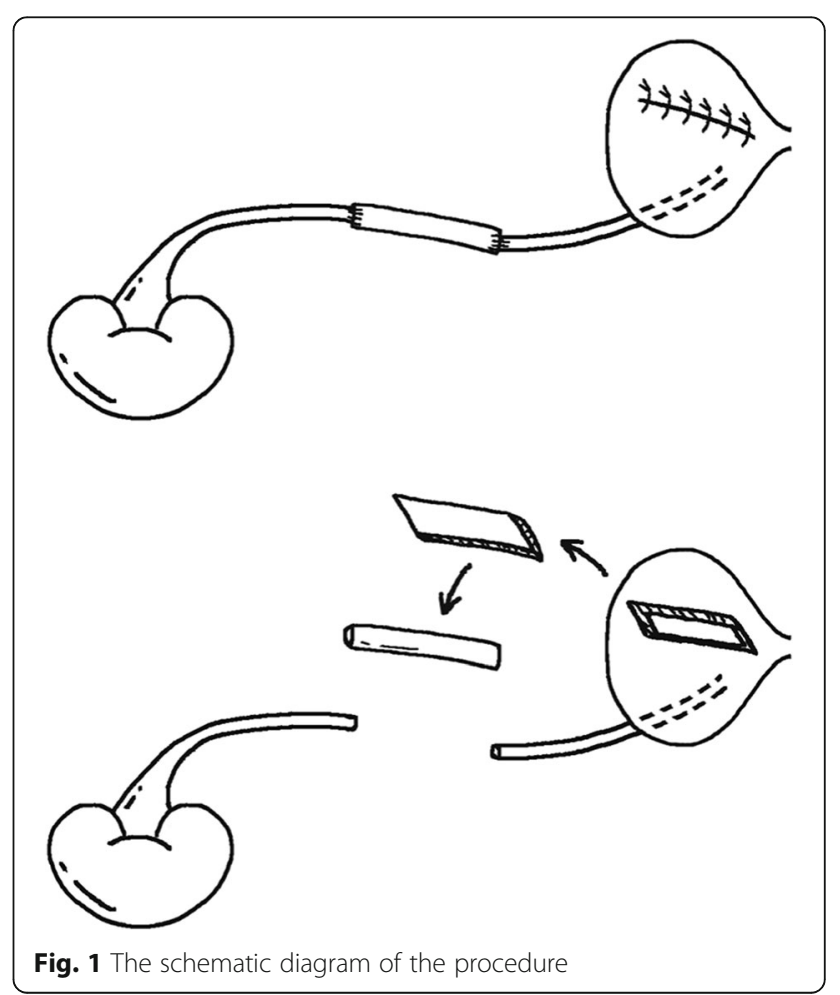




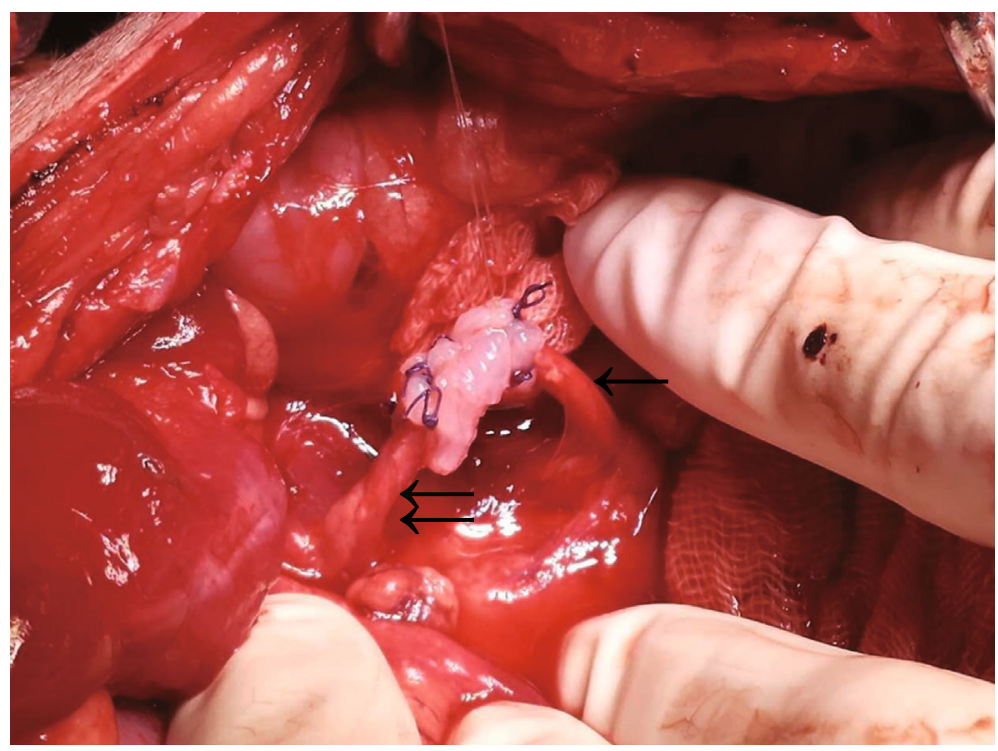

Fig. 2 A picture of the operation. Single arrow: upper ureter. Double arrow: lower ureter

\section{Results}

At the end point of the experiment, all the 9 dogs tolerated the procedure and survived. All had regular eating and activities. A subcutaneous abscess at the abdominal incision occurred to one dog, and was solved by a surgical debridement. There were otherwise no obvious signs of complications including severe gross hematuria, peritoneal infection or urine leakage. The ureteral stents in 7 animals were in place till the removal, and had been delivered out of the urinary tract in the other two. No obvious alteration of micturition rhythm was observed.

Through the abdominal exploration after the euthanasia, there was no hematoma, infection or urine leakage in the abdominal cavity. The reconstructive segments of the right ureters were intensely wrapped by fibrous adhesion. Mild hydronephrosis was observed in the right kidneys and upper ureters. Through the second cystostomy, urine was observed continuously outflowing from the orifices of the right ureters.

During the retrograde urography, the lumen of the neo-ureters easily accepted the 5-Fr tubes. The urography indicated normal ureteral caliber and patent urine excretion, without obvious fistula or stenosis. Grade of hydronephrosis assessed through the urography was accordant with the gross examination (Fig. 3).

The microscopic examination of the reconstructive ureteral segments showed that the open lumen was almost completely covered with pseudostratified urothelial lining. The urothelial cells formed the arrangement of 5-7 rows, similar to that of an empty bladder. In the denuding area, the basement membrane of urothelium was also complete. The submucosa and lamina muscularis were alive and healthy with abundant nutrient vessels.
Meantime, infiltration of inflammatory cells was observed in the submucosa. The smooth muscle fibers had disordered trends near the periureteral connective tissue, without serosa between the two layers. Nutrient vessels were also noticed in the periureteral connective tissue (Fig. 4).

\section{Discussion}

Ureteral injuries usually require surgical management, except for ureteral contusion and perforation. A short segment of ureteral defect can be repaired by end-to-

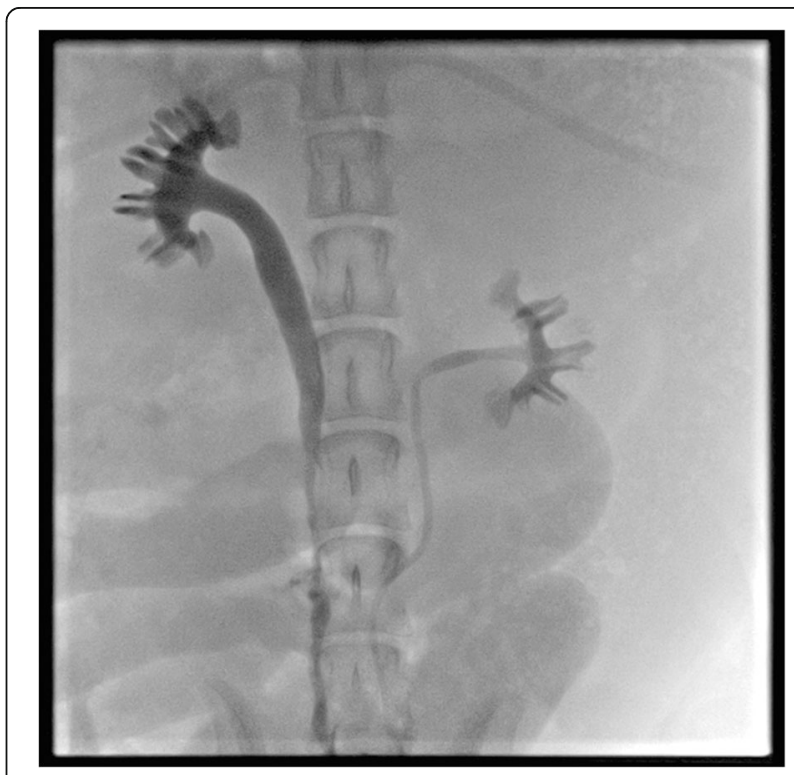

Fig. 3 The urography showing patent urine excretion and no fistula or stenosis (the left side as control) 

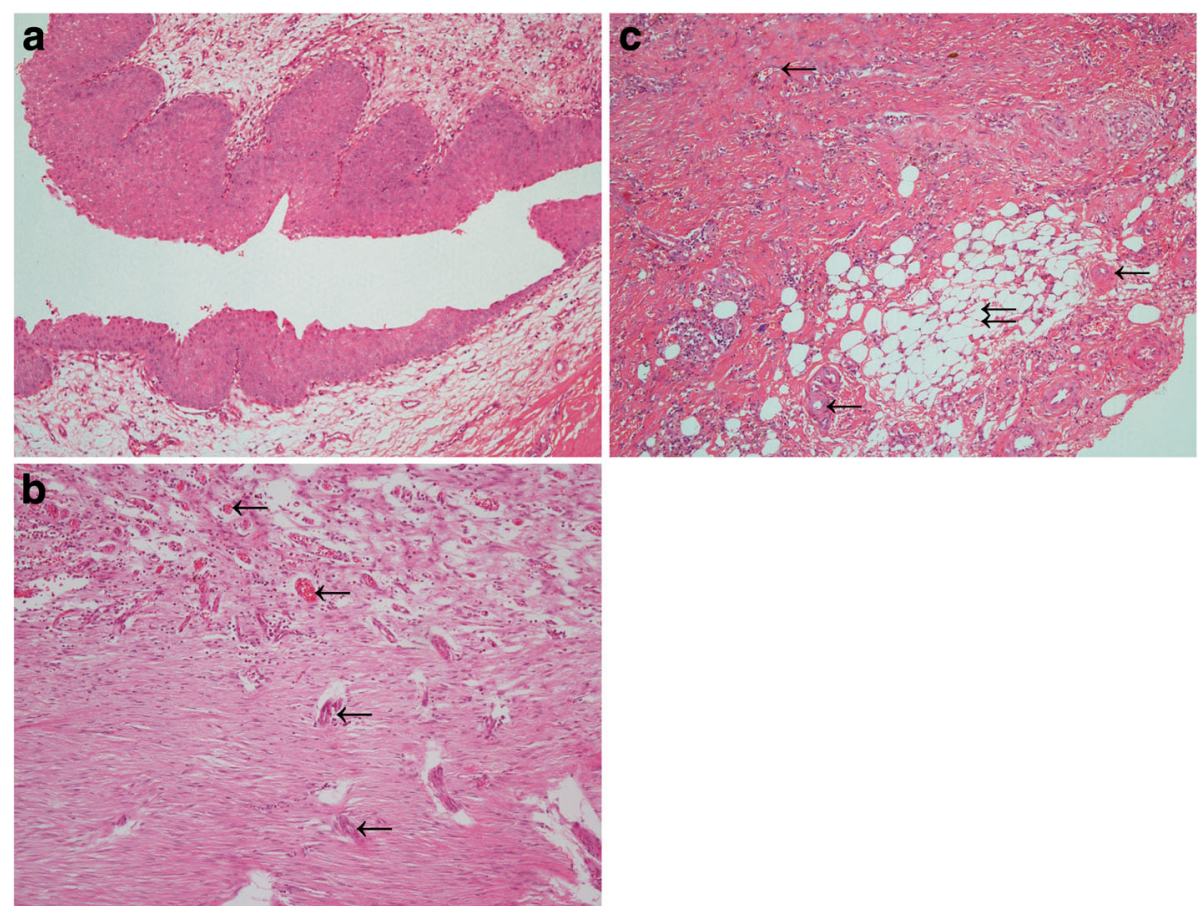

Fig. 4 Histological examination of reconstructive ureter (H.E.). a Longitudinal section view showing open lumen with complete urothelial lining in the neo-ureter. $(\times 10)$ b The healthy submucosa and lamina muscularis with nutrient vessels. $(\times 10)$ Single arrow: nutrient vessels in submucosa and lamina muscularis. c The disordered smooth muscle fibers, without a serosa between the lamina muscularis and connective tissue; nutrient vessels in the periureteral connective tissue. $(\times 10)$ Single arrow: The nutrient vessels of the reconstructive ureter. Double arrow: the periureteral connective tissue

end anastomosis, while a long-segment defect usually calls for advanced procedures and additional materials to reconstruct the continuity of injured ureter $[1,10]$. Tissue lined with urothelium may be the ideal material for ureteral reconstruction [1]. The reasons are associated with the characteristics of urothelial lining, including no reabsorption of ingredients of urine and no mucus secretion that may cause infection and lithogenesis. In brief, the urothelial lining can maintain the normal physiological functions of the ureter. The technique of a Boari flap is a classical example [3].

By contrast, intestinal or ileal substitution was regarded as the most accepted procedure for longsegment ureteral reconstruction so far. The advantages include adequate substitutive material and available blood supply for long-segment reconstruction [11]. However, it also involves numerous postoperative complications [12]. The absorptive and secretory functions of intestinal mucosa may lead to urine leakage, infection, anastomotic stenosis, lithogenesis, electrolyte and/or acid-base imbalance, and even renal function loss. The oversized caliber may exacerbate hydronephrosis and consequently accelerate impairment of renal function $[4-6,12]$. Interference to the gastrointestinal tract can also bring about postoperative problems [12]. The development of the Monti and Mitrofanoff techniques is an important improvement of intestinal substitution. Some previous studies claimed that the techniques were effective for ureteral replacement with sustained promising long-term results and consequent absence of metabolic complications $[7,8]$. On the other hand, some studies reported the postoperative intestinal complications such as ileus and leakage, which called for second operations sometimes [8, 13].

Novel methods using different materials for ureteral reconstruction have been developed, mostly avoiding involvement of intestinal mucosa. An intestinal seromuscular tunnel [1] and a seromuscular tapered ileal tube [10] were reported to form neo-ureter with complete urothelial lining of the inner surface. Previous studies demonstrated that it was feasible to rebuild normal urothelial lining of the reconstructive segment $[14,15]$. Moreover, an intestinal seromuscular segment with autograft of bladder mucosa was also proved applicable for ureteral reconstruction [16]. However, demucosalized intestinal segments may be complicated by shrinkage and regrowth of original gastrointestinal epithelium [17]. The efficacy of modified methods using intestinal segments demand further investigation. An autologous graft of granulation tissue capsule was also mentioned as a new material for ureteral substitution [12]. 
Recently, a spiral bladder muscle flap with vascular pedicles was designed to reconstruct full-length ureteral defects in 6 patients [2]. This procedure can avoid complex preoperative preparation and metabolic complications caused by intestinal substitution. It was claimed that the procedure can retain peristaltic function of bladder smooth muscle and consequently prevent dilation, fluid accumulation and lithogenensis. However, the authors also noted that kidney descent and fixation and a psoas hitch should be performed simultaneously to improve the success rate. Furthermore, preservation of superior vesical arteries required extremely subtle surgical techniques, which may lead to increased postoperative complications and limited popularization.

Considering its good compliance for the experimental surgery and resistance to infection, we selected beagles to establish the animal model. In our study, the gross examination and the urography identified that the neoureters were patent, only with mild hydronephrosis, which probably resulted from urine reflux due to the ureteral stent. The microscopic examination proved that the tapered bladder grafts were survivable without a vessel pedicle. Partial loss of urothelial lining, which was quite slight and local, may result from the retrograde catheterization and instillation of the contrast agent. The intact basement membrane allowed the healing of urothelium and thus avoided ureteral stenosis. Based on our histologic findings, we supposed that the nutrient vessels from periureteral connective tissue were sufficient to nourish the graft. Theoretically, the tapering procedure can reduce the volume of the graft tissue, and consequently decrease the nutrient demand. Meanwhile, it can create a raw surface of smooth muscle, cause inflammatory exudation and thus increase nutrient vessels and attachment between the graft and peripheral connective tissue. Our preliminary experience implied that a tapered non-vascularized bladder graft was a novel possible substitute material for ureteral reconstruction.

Another concern arose from the different capacity of bladder between the animal model and human beings. According to the previous studies, a 10-kg-heavy dog has a bladder capacity of approximately $40-70 \mathrm{ml}[18,19]$, while an adult human being has 300-500 ml. Comparatively, the beagles and human beings have a similar bladder capacity with respect to their own weight. Alteration of micturition rhythm was not observed in the animals, which indicated that the bladder capacity was not apparently influenced by the procedure. There was no concern about the application of our procedure for ureteral reconstruction in human beings.

The potential clinical application of this procedure is reconstruction of defect and stricture in upper and midureter, for which a psoas hitch or a Boari flap is unsuitable. The bladder has sufficient substituted material for required length of the neo-ureter. However, the primary limitations of our study were a small sample size and a short-term follow-up. Therefore, the efficacy and safety of this procedure must be confirmed by a larger sample size and longer postoperative follow-up assessments.

\section{Conclusion}

Reconstruction of ureteral defect and stricture maintains a troublesome problem for urologists. Additional to the traditional intestinal substitution, a number of new procedures and materials have been developed for ureteral reconstruction. Our findings showed that it was anatomically possible to use a tapered non-vascularized bladder graft for ureteral reconstruction in the experimental animal model. In consideration of the limitations of our study, further investigations with a larger sample size are expected to evaluate the long-term functional efficacy and safety.

\section{Acknowledgements \\ There were no particular people or institutions to be acknowledged during this study.}

\section{Funding}

This study was funded by the National Natural Science Foundation of China, No.81400705 and the National Basic Research Program of China (973 Program), No.2015CB943003.

\section{Availability of data and materials}

The data during the current study are available from the corresponding author on reasonable request.

\section{Authors' contributions}

$\mathrm{HJ}$ and QD conceived and designed the study. Surgical operation was conducted by SM, LZo, SL, LZh and HJ. Histological examination was done by $T Y$ and $Y H$. LZo drafted and wrote the manuscript, and HJ provided critical review. All authors read and approved the final manuscript.

Ethics approval and consent to participate

The protocol was reviewed and approved by the Huashan Institutional Review Board of Huashan Hospital, Fudan University. The 'Instructive Notions with Respect to Caring for Laboratory Animals' published in 2006 by the Science and Technology Department of China was followed strictly.

Consent for publication

Not applicable.

\section{Competing interests}

The authors declare that they have no competing interests.

\section{Publisher's Note}

Springer Nature remains neutral with regard to jurisdictional claims in published maps and institutional affiliations.

Received: 14 July 2016 Accepted: 16 October 2017

Published online: 23 October 2017

\section{References}

1. Hodjati H, Paidar JK, Kumar PV, Johari HG. Intestinal seromuscular tunneling: a novel method for ureteral replacement-an experimental design. Int Urol Nephrol. 2015:47(8):1351-5.

2. Li Y, Li C, Yang S, Song C, Liao W, Xiong Y. Reconstructing full-length ureteral defects using a spiral bladder muscle flap with vascular pedicles. Urology. 2014;83(5):1199-204. 
3. Stein R, Rubenwolf P, Ziesel C, Kamal MM, Thuroff JW. Psoas hitch and Boari flap ureteroneocystostomy. BJU Int. 2013;112(1):137-55.

4. Jednak R, Schimke CM, Barroso UJ, Barthold JS, Gonzalez R. Further experience with seromuscular colocystoplasty lined with urothelium. J Urol. 2000;164(6):2045-9.

5. Jednak R, Schimke CM, Ludwikowski B, Gonzalez R. Seromuscular colocystoplasty. BJU Int. 2001;88(7):752-6.

6. Kaefer M, Tobin MS, Hendren WH, Bauer SB, Peters CA, Atala A, Colodny AH, Mandell J, Retik AB. Continent urinary diversion: the Children's hospital experience. J Urol. 1997;157(4):1394-9.

7. Ali-el-Dein B, Ghoneim MA. Bridging long ureteral defects using the YangMonti principle. J Urol. 2003;169(3):1074-7.

8. Castellan M, Gosalbez R. Ureteral replacement using the Yang-Mont principle: long-term follow-up. Urology. 2006;67(3):476-9.

9. Bakari AA, Gadam IA, Aliyu S, Suleiman I, Ahidjo AA, Pindiga UH. Use of mitrofanoff and yang-monti techniques as ureteric substitution for severe schistosomal bilateral ureteric stricture: a case report and review of the literature. Niger J Surg. 2012;18(1):30-3.

10. Ibrahim ME, Ezzat MM, Ezzat WM. The use of seromuscular tapered ileal tube in ureteral replacement: an experimental model. Int Urol Nephrol. 2010;42(3):697-701.

11. Shokeir AA, Ghoneim MA. Further experience with the modified ileal ureter. J Urol. 1995;154(1):45-8.

12. Zhang J, GL G, Liu GH, Jiang JT, Xia SJ, Sun J, Zhu YJ, Zhu J. Ureteral reconstruction using autologous tubular grafts for the management of ureteral strictures and defects: an experimental study. Urol Int. 2012;88(1):60-5.

13. Maigaard T, Kirkeby HJ. Yang-Monti ileal ureter reconstruction. Scandinavian journal of urology. 2015;49(4):313-8.

14. Kuzaka B, Szymanska K, Borkowski A, Krus S. Restoration of the continuity of dog ureter after resection of its $5 \mathrm{~cm}$ middle segment. Br J Urol. 1996;77(3):342-6.

15. Kaefer M, Hendren WH, Bauer SB, Goldenblatt P, Peters CA, Atala A, Retik $A B$. Reservoir calculi: a comparison of reservoirs constructed from stomach and other enteric segments. J Urol. 1998:160(6 Pt 1):2187-90.

16. Zou J, Huang X, Su X, Lv D, Liao Y, Gong B, Qiu M. Experimental study on reconstruction of ureter by intestinal sero-muscular segment with autograft of bladder mucosa. Zhongguo xiu fu chong jian wai ke za zhi = Zhongguo xiufu chongjian waike zazhi $=$ Chinese journal of reparative and reconstructive surgery. 2010;24(5):594-8.

17. Heaney JA, Althausen AF, Parkhurst EC. lleal conduit undiversion: experience with tunneled vesical implantation of tapered conduit. J Urol. 1980;124(3): 329-33.

18. Geisse AL, Lowry JE, Schaeffer DJ, Smith CW. Sonographic evaluation of urinary bladder wall thickness in normal dogs. Vet Radiol Ultrasound. 1997; 38(2):132-7.

19. Atalan G, Barr FJ, Holt PE. Estimation of bladder volume using ultrasonographic determination of cross-sectional areas and linear measurements. Vet Radiol Ultrasound. 1998;39(5):446-50

\section{Submit your next manuscript to BioMed Central and we will help you at every step:}

- We accept pre-submission inquiries

- Our selector tool helps you to find the most relevant journal

- We provide round the clock customer support

- Convenient online submission

- Thorough peer review

- Inclusion in PubMed and all major indexing services

- Maximum visibility for your research

Submit your manuscript at www.biomedcentral.com/submit

) Biomed Central 